\title{
Chronic Abdominal Wall Pain Misdiagnosed as Functional Abdominal Pain
}

\author{
Tijmen van Assen, MD, Jenneke W. A.J. de Jager-Kievit, MD, \\ Marc R. Scheltinga, MD, PhD, and Rudi M. H. Roumen, MD, PhD
}

Introduction: The abdominal wall is often neglected as a cause of chronic abdominal pain. The aim of this study was to identify chronic abdominal wall pain syndromes, such as anterior cutaneous nerve entrapment syndrome (ACNES), in a patient population diagnosed with functional abdominal pain, including irritable bowel syndrome, using a validated 18-item questionnaire as an identification tool.

Methods: In this cross-sectional analysis, 4 Dutch primary care practices employing physicians who were unaware of the existence of ACNES were selected. A total of 535 patients $\geq 18$ years old who were registered with a functional abdominal pain diagnosis were approached when they were symptomatic to complete the questionnaire (maximum 18 points). Responders who scored at least the 10-point cutoff value (sensitivity, 0.94; specificity, 0.92) underwent a diagnostic evaluation to establish their final diagnosis. The main outcome was the presence and prevalence of ACNES in a group of symptomatic patients diagnosed with functional abdominal pain.

Results: of 535 patients, $304(57 \%)$ responded; 167 subjects $(31 \%)$ recently reporting symptoms completed the questionnaire. Of 23 patients who scored above the 10-point cutoff value, 18 were available for a diagnostic evaluation. In half of these subjects $(n=9)$ functional abdominal pain (including IBS) was confirmed. However, the other 9 patients were suffering from abdominal wall pain syndrome, 6 of whom were diagnosed with ACNES (3.6\% prevalence rate of symptomatic subjects; $95 \%$ confidence interval, 1.7-7.6), whereas the remaining 3 harbored a painful lipoma, an abdominal herniation, and a painful scar.

Conclusion: A clinically relevant portion of patients previously diagnosed with functional abdominal pain syndrome in a primary care environment suffers from an abdominal wall pain syndrome such as ACNES. (J Am Board Fam Med 2013;26:738-744.)

Keywords: Abdominal Pain, Anterior Cutaneous Nerve Entrapment Syndrome, Chronic Pain, Irritable Bowel Syndrome, Prevalence Studies

Diseased organs are frequently identified as the source of chronic abdominal discomfort and pain. If a patient's history, physical examination, and diagnostic evaluations are not abnormal, a common

This article was externally peer reviewed.

Submitted 11 April 2013; revised 27 June 2013; accepted 1 July 2013.

From the Department of Surgery, Máxima Medical Center, Veldhoven, The Netherlands (TvA, JWAJdJ-K, MRS, RMHR); and SolviMáx, Center of Excellence for Chronic Abdominal Wall and Groin Pain, Eindhoven, The Netherlands (MRS, RMHR).

Funding: none.

Conflict of interest: none declared.

Corresponding author: T. van Assen, Department of Surgery, Máxima Medical Center, de Run 4600, PO Box 7777, 5500 MB Veldhoven, The Netherlands (E-mail: tijmenvanassen@gmail.com). functional abdominal pain diagnosis, such as irritable bowel syndrome (IBS), which has a $10 \%$ to $15 \%$ prevalence in Europe and North America, ${ }^{1}$ may be considered. ${ }^{2}$ In contrast, the abdominal wall and pelvic floor often are neglected as a cause of chronic abdominal discomfort and pain. Apart from ignorance and the preoccupation of physicians with the viscera, complaints mimicking a problem of visceral origin (pseudo-visceral) as reported by patients also frustrate a diagnosis of chronic abdominal wall pain (CAWP). ${ }^{3-5}$

Myofascial pain and radiculopathy are rare examples of a CAWP syndrome. However, CAWP is commonly caused by the entrapment of an anterior cutaneous branch of one or more thoracic intercostal nerves. ${ }^{6}$ Patients with anterior cutaneous nerve 
entrapment syndrome (ACNES) have frequently undergone repetitive diagnostic evaluations. These tests are unfortunately almost always inconclusive, leading to a disillusioned patient and, even more so, a disappointed doctor. ${ }^{3,6-14}$ As a consequence, patients with CAWP often receive a diagnosis of exclusion such as IBS. ${ }^{2,15}$

The primary aim of this study was to identify patients with CAWP syndromes (in particular ACNES) in an IBS cohort in a primary care setting using a validated diagnostic, 18 -item abdominal wall pain questionnaire. We hypothesized that a portion of the population with IBS was incorrectly diagnosed and actually suffered from an abdominal wall pain syndrome. As a consequence of this study, awareness may be increased among primary care physicians.

\section{Methods}

This study was a cross-sectional analysis of primary care patients with IBS and was coordinated by members of the Surgical Department of the Máxima Medical Center (MMC) in Veldhoven/ Eindhoven, The Netherlands. The MMC Medical Ethical Committee approved the specifics of the study (no. NL36256.015.11). Sample size was estimated by a power analysis based on a common sense estimated prevalence prediction of $5 \%$ of patients with CAWP within an alleged IBS cohort, resulting in 292 subjects needed to screen. ${ }^{16}$ An estimated response rate of $60 \%$ would lead to a number needed to approach of 487 subjects.

A population with high risk of a CAWP syndrome within the IBS population was selected using a recently developed and validated 18 -item ACNES questionnaire (18-pAQ) (Figure 1). ${ }^{17}$ The 18-pAQ was found to clearly distinguish between abdominal wall pain syndromes such as ACNES and IBS. Scores of the 18-pAQ can range from 0 to 18 points. A 10 -point cutoff value was found to have an optimal 94\% sensitivity and $92 \%$ specificity.

Because our surgical department has a longstanding interest in abdominal wall pain syndromes that is frequently communicated to neighboring primary care physicians, it was decided to perform the study using patients from more distant practices. Therefore, 4 Dutch primary care practices located farther than $20 \mathrm{~km}$ from the MMC were asked to participate. Doctors participating in these
Figure 1. The typical position of anterior cutaneous nerve entrapment syndrome trigger points (Xs). At these points the anterior twigs of the intercostal nerves penetrate the rectus abdominal muscle (T712). The level of the umbilicus corresponds with intercostal nerve T10. The most typical site is T11 on the right side.

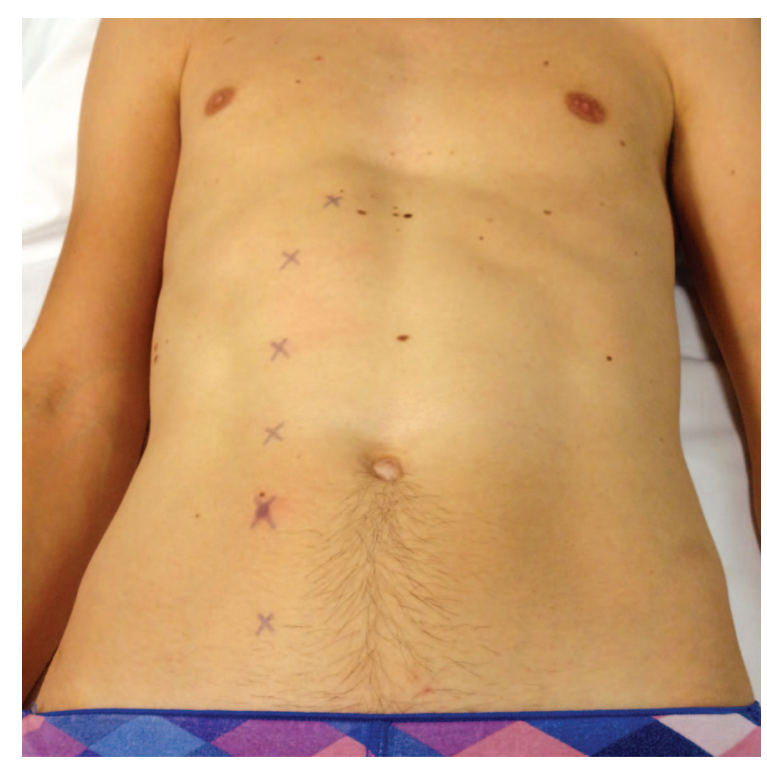

practices were unaware of ACNES. These selection criteria prevented bias and enhanced external validity before the start of this screening project.

For proper patient selection, participating practices were required to use an electronic patient record and to register according to the International Classification of Primary Care (ICPC) codes. This system is internationally accepted as a standard for coding and classifying complaints, symptoms, and diseases in primary care practices. The ICPC system consists of numbered chapters and sections. Chapter $\mathrm{D}$ focuses on the digestive tract. It contains 58 codes, ranging from malignant disease to functional disorders. ${ }^{18}$ The 8 codes associated with functional complaints demonstrate a spectrum of various complaints, but only 3 ICPC codes focus on complexes of symptoms: D01, generalized abdominal pain/cramps; D06, other localized abdominal pain; and D93, IBS. For the purpose of this study and the assumption of IBS as a diagnosis of exclusion, these latter 3 codes were used for patient selection. In the absence of a specific ICPC code for CAWP syndromes, we advise primary care physicians to use code D06 for this clinical entity. 
Figure 2. The trigger point is localized and palpated using a finger or a swab (left). Tenderness increases when abdominal muscle are tensed using Carnett's test (right).

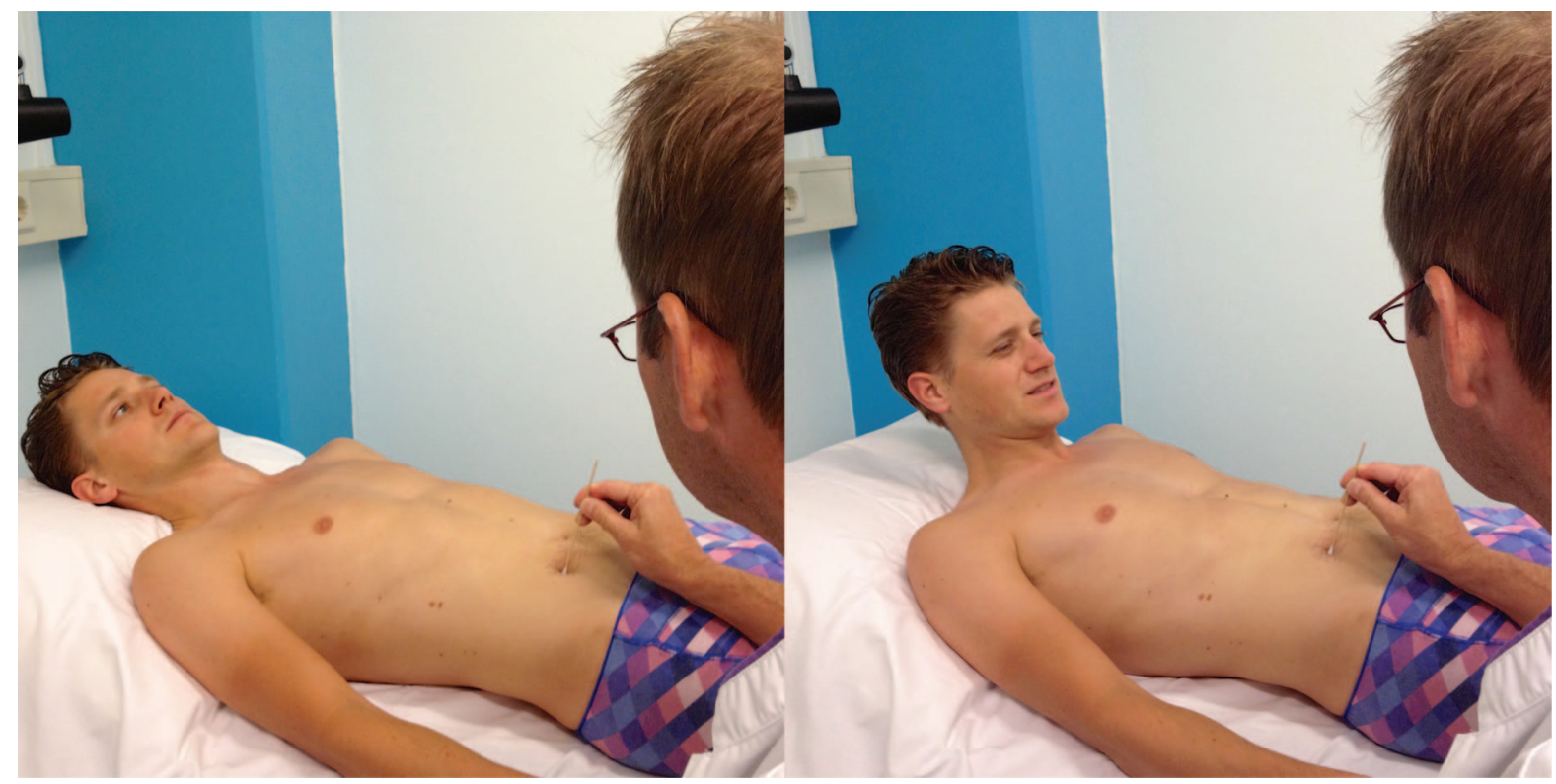

Patients $\geq 18$ years old who were previously registered with ICPC codes D01, D06, and D93 in these 4 selected practices were eligible for inclusion in the study. No limitations were applied regarding the moment of registration because of the chronic character of abdominal complaints. Charts of patients with a severe comorbidity causing immobility or additional gastrointestinal disease(s) were identified manually and excluded, as were patients with a language barrier. The remaining subjects were approached by their respective primary care physician via a letter of introduction. Subjects willing to participate were asked to complete the $18-\mathrm{pAQ}$. Nonsymptomatic subjects and subjects reporting other reasons not to participate were requested to return the nonparticipation form, which contained 2 options that could be selected: "no complaints anymore" and "other, namely. ..." Responses were registered and scored after 1 month. Returned and completed questionnaires with more than 1 missing item were excluded from evaluation.

Because of the logistic nature of the study and feasibility, subjects with a score of $<10$ points were not invited for further examination, possibly leading to a slight underestimation of the actual prevalence. Subjects with a score of $\geq 10$ points on the 18-pAQ were invited for a standardized history taking and physical examination by the first author (TvA). No gold standard is currently available for
ACNES (and other CAWP syndromes). An abdominal wall syndrome is essentially a clinical diagnosis that is suspected based on a triad of chronic localized abdominal pain within the lateral boundaries of the rectus abdominis muscle (Figure 2), a constant site of abdominal tenderness with a small $\left(<2 \mathrm{~cm}^{2}\right)$ area of maximal intensity (trigger point) and absence of abnormalities in previous blood analyses and imaging studies (colonoscopy, ultrasound, or computed tomography), if available. Carnett's test is a sensitive clinical test discriminating abdominal wall pain from visceral pain during a physical examination (Figure 3). ${ }^{7}$ The likelihood of ACNES as a typical example of a CAWP syndrome is increased when qualitative local somatosensory disturbances are present in proximity to the trigger point. ${ }^{19,20}$ All examination findings were registered on a case report form.

Data were analyzed using SPSS version 18.0 (IBM, Chicago, IL) for Mac OS X. Prevalence numbers were based on symptomatic responders and presented as percentages with associated standard deviations (SDs).

\section{Results}

A total of 583 patients were identified in the 4 selected primary care practices as dictated by the 3 ICPC codes of interest. After exclusion based on 
Figure 3. The validated questionnaire with the answer key. A 10-point cutoff value results in an optimal $94 \%$ sensitivity and $\mathbf{9 2} \%$ specificity.

\begin{tabular}{|c|c|c|c|}
\hline \multicolumn{4}{|c|}{18 item ACNES Questionnaire (18-pAQ) } \\
\hline Question & Mark the right answer & 1 Point if: & $0 / 1$ \\
\hline $\begin{array}{l}\text { 1. I experience bloating or a feeling } \\
\text { of gas in the intestines }\end{array}$ & Mostly / Regularly / Sometimes / Never & $\begin{array}{l}\text { Sometimes / } \\
\text { Never }\end{array}$ & \\
\hline $\begin{array}{l}\text { 2. Pain exists on different spots all } \\
\text { over the abdomen }\end{array}$ & Yes / No & No & \\
\hline $\begin{array}{l}\text { 3. For me PAIN dominates over } \\
\text { DISCOMFORT }\end{array}$ & Yes I No & Yes & \\
\hline $\begin{array}{l}\text { 4. Lying on the affected side } \\
\text { aggravates the pain }\end{array}$ & Mostly / Regularly / Sometimes / Never & $\begin{array}{l}\text { Mostly / } \\
\text { Regularly }\end{array}$ & \\
\hline $\begin{array}{l}\text { 5. The stool has an abnormal } \\
\text { consistency (e.g. hard and small, } \\
\text { pencil thin, loose, watery) }\end{array}$ & Mostly / Regularly / Sometimes / Never & $\begin{array}{l}\text { Sometimes / } \\
\text { Never }\end{array}$ & \\
\hline $\begin{array}{l}\text { 6. I believe the pain is originated } \\
\text { just beneath the skin }\end{array}$ & Yes / No & Yes & \\
\hline 7. Pain is sharp (cutting) & Mostly / Regularly / Sometimes / Never & $\begin{array}{l}\text { Mostly / } \\
\text { Regularly }\end{array}$ & \\
\hline $\begin{array}{l}\text { 8. I believe the complaints originate } \\
\text { from my gastrointestinal tract }\end{array}$ & Yes / No & No & \\
\hline $\begin{array}{l}\text { 9. I feel urgent need for bowel } \\
\text { movement without producing } \\
\text { stool (incomplete defecation) }\end{array}$ & Mostly / Regularly / Sometimes / Never & $\begin{array}{l}\text { Sometimes / } \\
\text { Never }\end{array}$ & \\
\hline $\begin{array}{l}\text { 10. Coughing, sneezing of squeezing } \\
\text { aggravates the pain }\end{array}$ & Mostly / Regularly / Sometimes / Never & $\begin{array}{l}\text { Mostly / } \\
\text { Regularly }\end{array}$ & \\
\hline $\begin{array}{l}\text { 11.Pain is always located on the } \\
\text { same spot(s) }\end{array}$ & Yes / No & Yes & \\
\hline $\begin{array}{l}\text { 12.Pain is located just lateral to the } \\
\text { midline of the abdomen }\end{array}$ & Yes / No & Yes & \\
\hline $\begin{array}{l}\text { 13. Complaints are somehow related } \\
\text { to an altered defecation pattern }\end{array}$ & Yes / No & No & \\
\hline $\begin{array}{l}\text { 14.Pain is provoked by daily } \\
\text { activities (e.g. walking, sitting, } \\
\text { cycling, bending) }\end{array}$ & Mostly / Regularly / Sometimes / Never & $\begin{array}{l}\text { Mostly / } \\
\text { Regularly }\end{array}$ & \\
\hline $\begin{array}{l}\text { 15. The painful spot feels strange, } \\
\text { different or dull }\end{array}$ & Mostly / Regularly / Sometimes / Never & $\begin{array}{l}\text { Mostly / } \\
\text { Regularly }\end{array}$ & \\
\hline 16. Stress provokes the complaints & Mostly / Regularly / Sometimes / Never & \begin{tabular}{|l|} 
Sometimes / \\
Never
\end{tabular} & \\
\hline $\begin{array}{l}\text { 17. The most intense pain can be } \\
\text { localized by the tip of one finger }\end{array}$ & Yes / No & Yes & \\
\hline $\begin{array}{l}\text { 18.Pushing on the painful spot } \\
\text { aggravate the complaints }\end{array}$ & Mostly / Regularly / Sometimes / Never & $\begin{array}{l}\text { Mostly / } \\
\text { Regularly }\end{array}$ & \\
\hline & & Total: & \\
\hline
\end{tabular}

age $<18$ years $(n=6)$, comorbidity $(n=13)$, other known gastrointestinal disease $(\mathrm{n}=8)$, absence of complaints $(\mathrm{n}=2)$ and personal reasons $(\mathrm{n}=19)$, 535 patients were eligible for screening. Some 369 patients were registered with ICPC code D93 (IBS). The remaining subjects $(\mathrm{n}=166)$ were diagnosed with generalized or localized abdominal pain (codes D01 and D06).

The response rate was $57 \%(\mathrm{n}=304)$. Of this responding population, we analyzed the questionnaires of 167 subjects signing informed consent (68\% women, age $54 \pm 17$ years). The remaining 137 subjects did not participate in screening (no complaints at present, $\mathrm{n}=94$; personal reason, $\mathrm{n}=$ 37; missing items in the $18-\mathrm{pAQ}, \mathrm{n}=6$ ).

Of these 167 patients, 23 scored $\geq 10$ points on the 18-pAQ. After being contacted, 18 individuals were willing to undergo history taking and physical examination. Nine subjects were found to have IBS, generalized abdominal pain/cramps, or other localized abdominal pain, as diagnosed previously. The other half of this group $(n=9)$ was diagnosed with a CAWP syndrome, 6 of whom had ACNES (code D93, $\mathrm{n}=4$; code D01, $\mathrm{n}=2$ ). The remaining 3 patients were diagnosed with a painful lipoma $(\mathrm{n}=$ $1)$, an abdominal wall herniation $(\mathrm{n}=1)$, and scar tissue pain $(\mathrm{n}=1)$ based on physical examination and ultrasound investigation (Figure 4).

Of the 6 identified patients with ACNES, 4 received a subfascial trigger point injection using $1 \%$ lidocaine, 3 of whom experienced a long-lasting and satisfactory reduction in pain ( $>3$ months). The fourth patient with ACNES received additional manual therapy because the pain was refractory to injections. A neurectomy will be considered if she remains unresponsive. ${ }^{14,20}$ Pain levels of patients 5 and 6 were mild. These 2 individuals were reassured once the origin of the pain was explained. 
Figure 4. Flow chart of the study protocol. ACNES, anterior cutaneous nerve entrapment syndrome; CAWP, chronic abdominal wall pain; $\mathbf{G I}$, gastrointestinal.

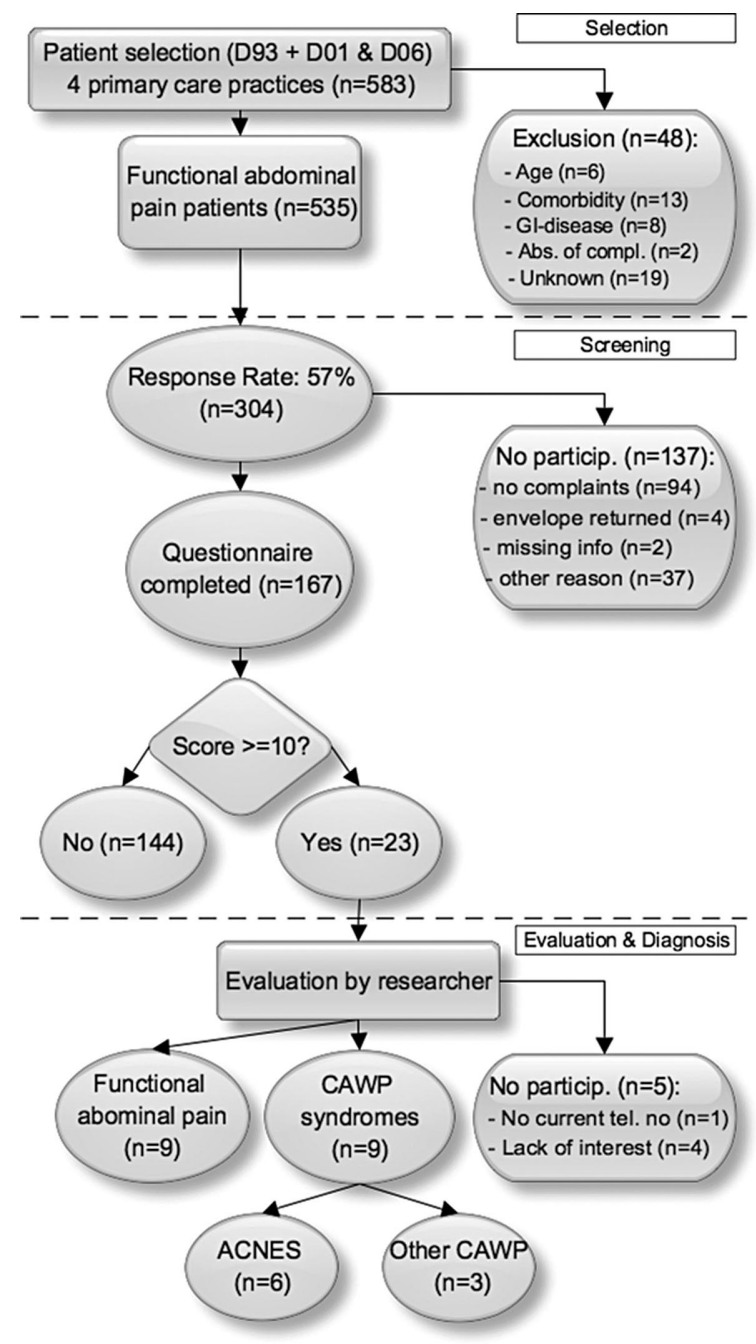

Despite the somewhat smaller sample size than calculated in the power analysis, the prevalence of ACNES within the symptomatic functional abdominal pain population in these 4 selected primary care practices was calculated to be 3.6\% (95\% confidence interval [CI], 1.7-7.6).

\section{Discussion}

This study demonstrates that ACNES may be diagnosed in some patients currently being treated for or diagnosed with functional abdominal complaints such as IBS. Six subjects harbored characteristics that are typically associated with ACNES, corresponding to a $3.6 \%$ prevalence $(95 \%$ CI, $1.7-$ 7.6) in the responding population with alleged IBS $(\mathrm{n}=167)$. This percentage is based on symptomatic responders. Although this may seem a relatively small number, a substantial portion of the Dutch population is registered as having functional abdominal complaints. Estimations of the prevalence of IBS in the Netherlands may vary from $10 \%$ to $20 \%$ (of a population of 16.7 million $^{21}$ ). Considering that 1 in 3 people with complaints related to IBS seek medical help, we calculate that a minimum of half a million individuals consult a doctor for this problem in the Netherlands. ${ }^{22-25}$ On the basis of these assumptions, some 20 thousand Dutch individuals with ACNES are currently misdiagnosed as having functional abdominal pain (95\% CI, 9.500-42.000). Using similar assumptions and calculations, some 400,000 US individuals with functional abdominal syndromes may suffer from an undiagnosed CAWP such as ACNES. ${ }^{26,27}$

During the study's analysis period, a seventh patient with ACNES was identified by one of the participating primary care physicians, who was now familiar with the typical diagnostic triad of ACNES. Remarkably, this patient was registered with an ICPC code belonging to the nervous system chapter, probably because of predominant somatosensory alterations of the abdominal skin. This patient with newly diagnosed CAWP illustrates the diversity of interpretations by primary care physicians who are unaware of ACNES. However, an important observation of this study is the immediate effect of heightened awareness among primary care physicians regarding CAWP syndromes.

A survey of Dutch surgeons and residents confirmed a hypothesized ignorance of ACNES among hospital-based specialists. More than $85 \%$ of the respondents confessed they had never heard of this syndrome (response rate, 35\% [507 of 1450]). Moreover, only $18 \%$ of senior surgeons ( $>10$ years of experience) were aware of this clinical entity. ${ }^{28} \mathrm{It}$ is not surprising that doctors' delay in diagnosing CAWP syndromes is substantial and many patients with ACNES remain undiagnosed. This situation is not only a Dutch issue; CAWP is a globally undervalued medical problem and a diagnostic challenge. Therefore, clinicians worldwide require education on abdominal wall pain problems so that these clinical entities will be identified at an earlier stage and treated accordingly. A complicating factor is that ACNES patients often report pseudo-visceral 
complaints seemingly unrelated to an abdominal wall problem (bloating, nausea, or pain related to, eg, food intake, altered defecation pattern, weight loss). ${ }^{4,5}$

The 18-pAQ diagnostic questionnaire was found to harbor good discriminative properties during the validation study. ${ }^{17}$ In a population with low prevalence, such as the current one, a $50 \%$ positive predicted value is suboptimal but acceptable. As a comparison, a national screening program for breast cancer in the Netherlands has a positive predictive value of $41 \% .{ }^{29}$ However, one can question whether this tool is performing appropriately in the hands of physicians with little knowledge of CAWP syndromes. Considering the suboptimal sensitivity of the questionnaire, it is likely that some patients with CAWP in the investigated cohort remained unidentified. However, a subpopulation may be identified (approximately $10 \%$ to $15 \%$ of the total population) by the questionnaire and invited for specific examination.

This study has shortcomings. A flaw is the relatively low response rate of subjects completing the 18 -pAQ, resulting in a prevalence with a wide CI. One explanation is that approximately a third of all responders $(31 \%)$ claimed to have no complaints at present and therefore did not complete the questionnaire. Moreover, the largest group of nonparticipants did not respond at all (43\%). Patients seeking help probably are more likely to respond. Furthermore, all patients who were ever diagnosed with IBS or localized/generalized abdominal pain were eligible for the study. This inclusion criterion was deemed important because the intensity of symptoms of both IBS and ACNES may fluctuate over time. A low response rate among patients with few symptoms at the time of questionnaire reception is therefore likely. Another point of concern is a possible flaw in the ICPC code registration process. Under-registration and updating of patients' files may be suboptimal in a demanding practice.

What is the clinical relevance of identifying patients with ACNES in a group in which the majority of patients have IBS? On the one hand, patients diagnosed with ACNES (preferably at an early stage) are likely to undergo fewer unnecessary medical procedures and may benefit from a relatively simple treatment regimen. A treatment stratagem including injections or surgery resulted in total or substantial long-term pain relief in $80 \%$ of patients with ACNES. ${ }^{14}$ On the other hand, even after unsuccessful treatment of IBS, an alternative diagnosis is not easily considered. This is especially because of the low effectiveness of treatment for IBS ( $<60 \%$ benefits from treatment with antispasmodics and antidepressants ${ }^{30}$ ). Patients diagnosed incorrectly with abdominal wall pain most probably do not experience any effect of these IBS treatment strategies. Moreover, a proper and effective treatment regimen is not instituted, leading to prolonged suffering.

\section{Conclusion}

A clinically relevant portion of patients incorrectly diagnosed with a functional abdominal pain syndrome within the primary care setting suffers from a CAWP syndrome, including ACNES. Once correctly diagnosed, treatment is simple and effective. Increased awareness of CAWP syndromes may have a considerable effect on an individual level as well as on the level of the health care system.

\section{References}

1. WGO practice guideline. Irritable bowel syndrome: a global perspective. Milwaukee, WI: World Gastroenterology Organization; 2009. Available from: http:// www.worldgastroenterology.org/irritable-bowelsyndrome.html. Accessed September 9, 2013.

2. Spiegel BM, Farid M, Esrailian E, Talley J, Chang L. Is irritable bowel syndrome a diagnosis of exclusion?: a survey of primary care providers, gastroenterologists, and IBS experts. Am J Gastroenterol 2010;105: 848-58.

3. Sharpstone D, Colin-Jones DG. Chronic, non-visceral abdominal pain. Gut 1994;35:833-6.

4. Choi YK, Chou S. Rectus syndrome. Another cause of upper abdominal pain. Reg Anesth 1995; 20:347-51.

5. Haynsworth RF Jr, Noe CE. An unusual presentation of intercostal neuralgia. Anesthesiology 1990; 73:779-80.

6. Srinivasan R, Greenbaum DS. Chronic abdominal wall pain: a frequently overlooked problem. Practical approach to diagnosis and management. Am J Gastroenterol 2002;97:824-30.

7. Carnett J. Intercostal neuralgia as a cause of abdominal pain and tenderness. Surg Gynecol Obstet 1926; 42:8.

8. Applegate WV. Abdominal cutaneous nerve entrapment syndrome. Surgery 1972;71:118-24.

9. Hershfield NB. The abdominal wall. A frequently overlooked source of abdominal pain. J Clin Gastroenterol 1992;14:199-202.

10. Greenbaum DS, Greenbaum RB, Joseph JG, Natale JE. Chronic abdominal wall pain. Diagnostic validity and costs. Dig Dis Sci 1994;39:1935-41. 
11. Abdominal wall tenderness test: could Carnett cut costs? Lancet 1991;337:1134.

12. Suleiman S, Johnston DE. The abdominal wall: an overlooked source of pain. Am Fam Phys 2001;64: 431-8.

13. Applegate WV. Abdominal cutaneous nerve entrapment syndrome (ACNES): a commonly overlooked cause of abdominal pain. Perm J 2002;6:20-7.

14. Boelens OB, Scheltinga MR, Houterman S, Roumen RM. Management of anterior cutaneous nerve entrapment syndrome in a cohort of 139 patients. Ann Surg 2011;254:1054-8.

15. Rome Foundation [homepage]. Available from: http://www.romecriteria.org/. Accessed September 9, 2013.

16. Naing L. Practical issues in calculating the sample size for prevalence studies. Arch Orofac Sci 2006;1: 9-14.

17. van Assen T, Boelens OB, Kamphuis JT, Scheltinga MR, Roumen RM. Construction and validation of a questionnaire distinguishing a chronic abdominal wall pain syndrome from irritable bowel syndrome. Frontline Gastroenterol 2012;3:288-94.

18. Njoo K. ICPC. 2012. Available from: https://www. nhg.org/themas/artikelen/icpc. Accessed November 1, 2009.

19. Boelens OB, Scheltinga MR, Houterman S, Roumen RM. Randomized clinical trial of trigger point infiltration with lidocaine to diagnose anterior cutaneous nerve entrapment syndrome. Br J Surg 2013;100: 217-21.

20. Boelens OB, van Assen T, Houterman S, Scheltinga MR, Roumen RM. A double-blind, randomized, controlled trial on surgery for chronic abdominal pain due to anterior cutaneous nerve entrapment syndrome. Ann Surg 2013;257:845-9.

21. Centraal Bureau voor de Statistiek. Population counter [web page, in Dutch]. Available from: http:// www.cbs.nl/nl-nl/menu/themas/bevolking/cijfers/ extra/bevolkingsteller.htm. Accessed September 9, 2013.

22. Drossman DA, Whitehead WE, Camilleri M. Irritable bowel syndrome: a technical review for practice guideline development. Gastroenterology 1997;112: $2120-37$.

23. Talley NJ, Weaver AL, Zinsmeister AR, Melton LJ 3rd. Onset and disappearance of gastrointestinal symptoms and functional gastrointestinal disorders. Am J Epidemiol 1992;136:165-77.

24. Van der Velden J. [A national study for diseases and procedures in the primary care practice. Base report; Morbidity in the primary care practice]. NIVEL, 1991.

25. Okkes IM, Oskam SK, Lamberts H. [From complaint to diagnosis.] Bussum: Coutinhor; 1998.

26. Saito YA, Schoenfeld P, Locke GR 3rd. The epidemiology of irritable bowel syndrome in North America: a systematic review. Am J Gastroenterol 2002; 97:1910-5.

27. U.S. and world population clock. Washington, DC: US Census Bureau. Available from: http:// www.census.gov/main/www/popclock.html. Accessed September 9, 2013.

28. Roumen RM. and Scheltinga MR. [Abdominal intercostal neuralgia: a forgotten cause of abdominal pain]. Nederlands tijdschrift voor geneeskunde 2006; 150(35): p. 1909-15.

29. CBO, K.v.d.g., [Guideline Breast cancer, N.B.O. Netherlands, Editor 2008, Quality institute for health care $\mathrm{CBO}$ ]: Utrecht. p. 274.

30. Ruepert L, Quartero AO, de Wit NJ, van der Heijden GJ, Rubin G, Muris JW. Bulking agents, antispasmodics and antidepressants for the treatment of irritable bowel syndrome. Cochrane Database Syst Rev 2011;(8):CD003460. 